\title{
Analytical and Comparative Study of Different Types of Two-Leg Chopping up Regulator
}

\author{
Walid Emar ${ }^{1}$ \\ Electrical Engineering Department \\ Isra University, Faculty of Engineering \\ Amman, 11622 Jordan
}

\author{
Omar A. Saraereh ${ }^{2}$ \\ Electrical Engineering Department \\ The Hashemite University, Faculty of Engineering \\ Zerqa, 13133 Jordan
}

\begin{abstract}
The main focus of this article is to analyze and simulate the two-leg parallel connection of a chopping up regulator with flattering inductive smoothers or with an interphasing centre-tap transformer supplied by a three-phase diode rectifier and a DC link in between. The article deals with the problem of reducing total harmonic distortion, minimizing THD and EMI with low switching frequency. The Simulated three phase a.c. load model is added at the end to investigate the current and voltage harmonics. The main objective of this paper is the investigation of the problem and active impact of replacing flattering inductive smoothers used to reduce voltcurrent fluctuating waveforms of the chopping up regulator by new topology known as interphasing centre-tap transformer with magnetic coupling. The comparison of these two variations of the study is then done based on their technical parameters and economical investment viewpoint. The considered technical parameters are to be current distribution into individual legs, amount of voltcurrent ripple and area of discontinuous currents. The investment costs governed by the material requirements are essential for transformer and smoother production design. The outcome of using the interphasing centre-tap transformer is successive cancelation of voltcurrent fluctuating waveforms generated at the output of the chopping up regulator. This is proved by an experiment with 35 in input and power chopping up400-V/90-A.Software simulations in Simplorer and Matlab/Simulink or software program and regimen prototypes have been arranged to confirm the results.
\end{abstract}

Keywords-Chopping up regulator with a flattering inductive smoother; magnetic coupling; connection with interphasing centretap transformer

\section{INTRODUCTION}

In many manufacturing applications it is necessary to change the dc direct voltcurrent source into a dc variable one. A chopping up regulator directly changes from dc to dc and like an ac transformer, it is used to step up or step down a dc voltage source value. Due to its capability to deliver smoothly adjustable dc voltage, dc chopper ups have revolutionized the regime industrial control devices and frequency inverters with unidirectional and with power levels extending from fractional horsepower up to several megawatts $[1,2]$.

The chopping up regulator is required for the use in traction motor control applications in electric cars, marine vehicles, marine lifts and forklift. The chopping up regulator moreover can be used in braking regime of motors to return back to the source energy which results in energy savings for transport systems with regular stops. In addition, the Chopper ups are used together with a capacitive filter respectively with an inductive smoother to generate dc voltage respectively direct current sources.

Regulated inverters are widely used these days to control the voltage at the output of solar systems. Another form for stepping up the voltage would be a PWM inverter followed by a chopping up regulator [3].

Due to the periodic chopper up function, its current waveforms and its output voltage contain certain harmonics [4]. This type of harmonics has an impact on load performance, chopper up losses and rises the range of discontinuous (interrupted) currents in which the chopper up can operate, and as a result, its operation is more complex and the control characteristics will in many cases be changed which requires additional control system unitsand special controllers.

In practice, inductive smoothers are used to lower the harmonic level, but the magnitude of these inductive smoothers increases with increasing input and output curve fluctuations $[5,6]$.

To improve the performance of chopping up regulators in reducing variable waves generated at its input or output, the chopping up regulator structure is usually improved by adding two or more legs to act in parallel. The outputs of these individual legs are combined by inductive smoothers without magnetic coupling. Generally, the switching technique of these legs introduces a phase displacement of the control signals between the individual legs and at the same time keeps them working with the same switching frequency. This type of connection is referred to as a several-leg parallel connection of a chopping up regulator with flattering inductive smoothers [7-9].

A better solution is attained if a magnetic coupling of each two flattering inductive smoothers is introduced. This new topology of flattering inductive smoother is recognized as an interphasing centre-tap transformer and the connection of chopping up regulators in this case is known as a several-leg parallel connection of chopping up regulators with an interphasing centre-tap transformer. In this case the number of legs must be even.

The result is a successive cancellation of the voltcurrent curves fluctuations with little design demands on the smoothers. It also makes the control of the regulator easier 
since there will be no need for additional controllers to equally divide the currents into the individual legs. The magnetic coupling between each two smoothers takes care of that [1012].

In this paper, an analysis of an interleaved chopping up regulator supplied from an ac network via a single phase bridge rectifier circuit is carried out. An interleaved converter with two-leg converters connected in parallel was considered for this work. The performance of the conventional chopping up regulator, two-leg interleaved chopping up regulator with inductive smoothers or with an interphasing centre-taped transformer were compared by means of simulation and design. Simulation of the system was carried out using MATLAB/Simulink. A design example is presented to illustrate the novel design concept. The formatter will need to create these components, incorporating the applicable criteria that follow.

\section{BASIC CONFIGURATION OF CHOPPING UP REGULATOR}

Fig. 1 shows the topology of a fundamental connection of a chopping up regulator. The main parts are an a.c. power supply via a single phase bridge rectifier, input capacitive filter (capacitance $\mathrm{C}_{\mathrm{f}}$ with inductance $\mathrm{L}_{\mathrm{f}}$ ) flattering inductive smoother, L, power electronic switch (BJT, IGBT, MOSFET or THYRISTOR), diode, and output capacitive filter. The load is considered to be a resistor of resistance $R_{a}$ with an smoother of inductance $\mathrm{L}_{\mathrm{a}}$.

The principle of operation of an elementary chopping up regulator shown in Fig. 1(a) is currently known. A chopping up regulator varies its average output voltage $V_{o}$ that appears across the load which is relative to its input $V_{s}$, by varying the proportion of its operating time during which the output is connected to the input. In other words, the unspecified switching device $\mathrm{S}$ operates with a regular periodic time $T$, and is closed for a time $t_{o n}=T-t_{o f f}$.

\section{A. Continuoius Operating Regime}

The regulator simulation parameters for the current continuous regime are listed in Table I as:

When the switch is closed, the diode is reverse biased. Kirchhoff's voltage law around the path containing the source, smoother $\mathrm{L}$, and closed switch $\mathrm{S}$ is:

$\mathrm{V}_{\mathrm{s}}=\mathrm{L} \frac{\mathrm{di}_{\mathrm{L}}}{\mathrm{dt}}=\mathrm{V}_{\mathrm{L}}$

The peak to peak current ripple of the smoother current is then computed from:

$\Delta i_{L}=\frac{V_{S}}{L} t_{o n}=\frac{V_{S}}{f L} k, k=\frac{t_{o n}}{T}$

$\mathrm{k}$ is a duty time ratio of the chopping up regulator. When the switch is opened, due to the energy stored in the smoother $\mathrm{L}$, the source current, cannot change instantaneously, so the diode becomes forward-biased and conducts the source current. Assuming that the load voltage $V_{o}$ is

$V_{s}-V_{o}=L \frac{d i_{L}}{d t}=v_{L} \Rightarrow \Delta i_{L}=\frac{V_{o}-V_{s}}{f L} t_{o f f}=\frac{V_{o}-V_{S}}{f L}(1-k)$
TABLE I. TeChNicAl PARAMETERs OF THE Simulated REgUlatoR

\begin{tabular}{|l|l|l|l|}
\hline Parameter & Symbol & Real value \\
\hline Smoothing filter: & $L$ & $30 \mathrm{mH}$ \\
\hline Regulator operating period: & $T$ & $20 \mathrm{~ms}$ \\
\hline Supply voltage & $V_{s}$ & $35 \mathrm{~V}$ \\
\hline Load resistance: & $R_{\mathrm{a}}$ & $5 \Omega$ \\
\hline Load inductance: & $L_{a}$ & $2 \mathrm{mH}$ \\
\hline Output capacitive filter & $C$ & $5 \mathrm{mF}$ \\
\hline
\end{tabular}

Where $\Delta i_{L}$ is the peak to peak current ripple in the smoother current, when the switch is open. Under steady-state operation conditions, the net change in smoother voltage must be zero. Using Eqs. (2) and (3), it yields [2]:

$V_{o}=\frac{V_{s}}{1-k} \Rightarrow k=1-\frac{V_{s}}{V_{o}}$

Substituting from Eq. 4 into Eq. 2 or Eq. 3, results in the following value for the smoother current ripple:

$\Delta i_{L}=\frac{V_{o}-V_{S}}{f L}(1-k)$

By expressing the operating period of the converter as $=t_{o n}+t_{o f f}$, then from Eqs. 2 and 3, we may describe the current ripple of the smoother $i_{L}$ or the source current $i_{S}$ as a function of the output load voltage as follows:

$\Delta i_{L}=\frac{V_{S}\left(V_{o}-V_{S}\right)}{f L V_{o}}$

The maximum value of $\Delta i_{L}$ as a function of the output load voltage $V_{o}$ occurs when $V_{o}$

$\Delta i_{\text {Lmax }}=\frac{V_{o}}{f L}$

\section{B. Discontinuous Operating Regime}

The chopping up regulator may also operate in discontinuous operating regime due to small values of the smoother inductance $(L=1 \mathrm{mH})$ or the use of low frequency switches [16].

In some cases, the discontinuous smoother current, is desirable for control reasons when the output is regulated. The smoother current and load voltage ripples are determined from the fact that the average smoother voltage is zero.

The source and diode currents for discontinuous current regime have the basic waveforms as shown in Fig. 1(c). When the main switch, $\mathrm{S}$, is on, the voltage across smoother, $\mathrm{L}$, is $V_{s}$. But when the switch is off and the smoother current, is decreasing, the smoother voltage is $V_{s^{-}} V_{o}$. The smoother current, falls down until it touches the zero-axis before the end of the switch operating period, T. With the switch and diode are off, the smoother current, is zero. The average voltage across the smoother is:

$V_{s} k+\left(V_{s}-V_{o}\right) k_{1}=0 \Rightarrow V_{o}=V_{s}\left(\frac{k}{k_{1}}+1\right)$ 
Where $k_{1}=t_{k} / T, t_{k}$ is the instant of interruption at which the smoother current reaches its zero value when the diode becomes off. The maximum value of the discontinuous smoother current is the same as the peak to peak current ripple in this current, when the switch is closed:

$I_{L \max }=\frac{V_{S}}{f L}\left(1-\frac{V_{S}}{V_{o}}\right)$

3PHAS

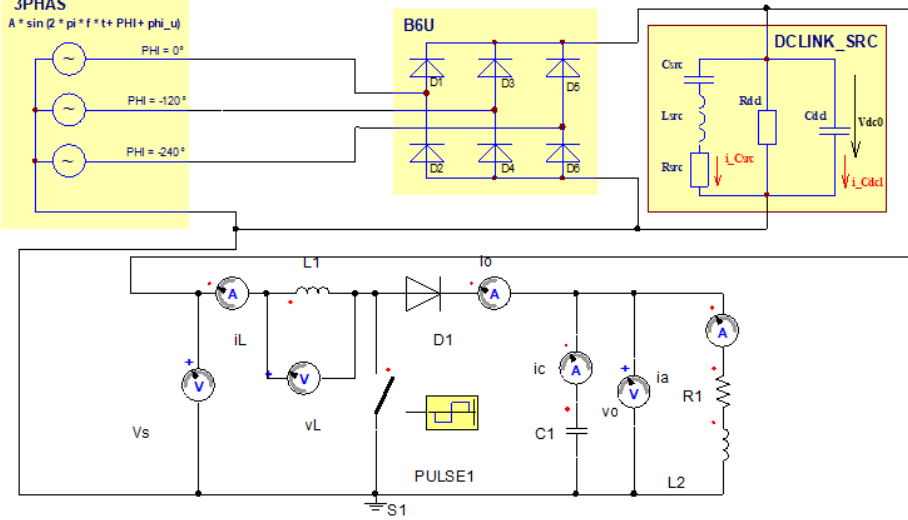

(a) Basic Chopping up Regulator Supplied from an ac Network via a Rectifier Circuit.

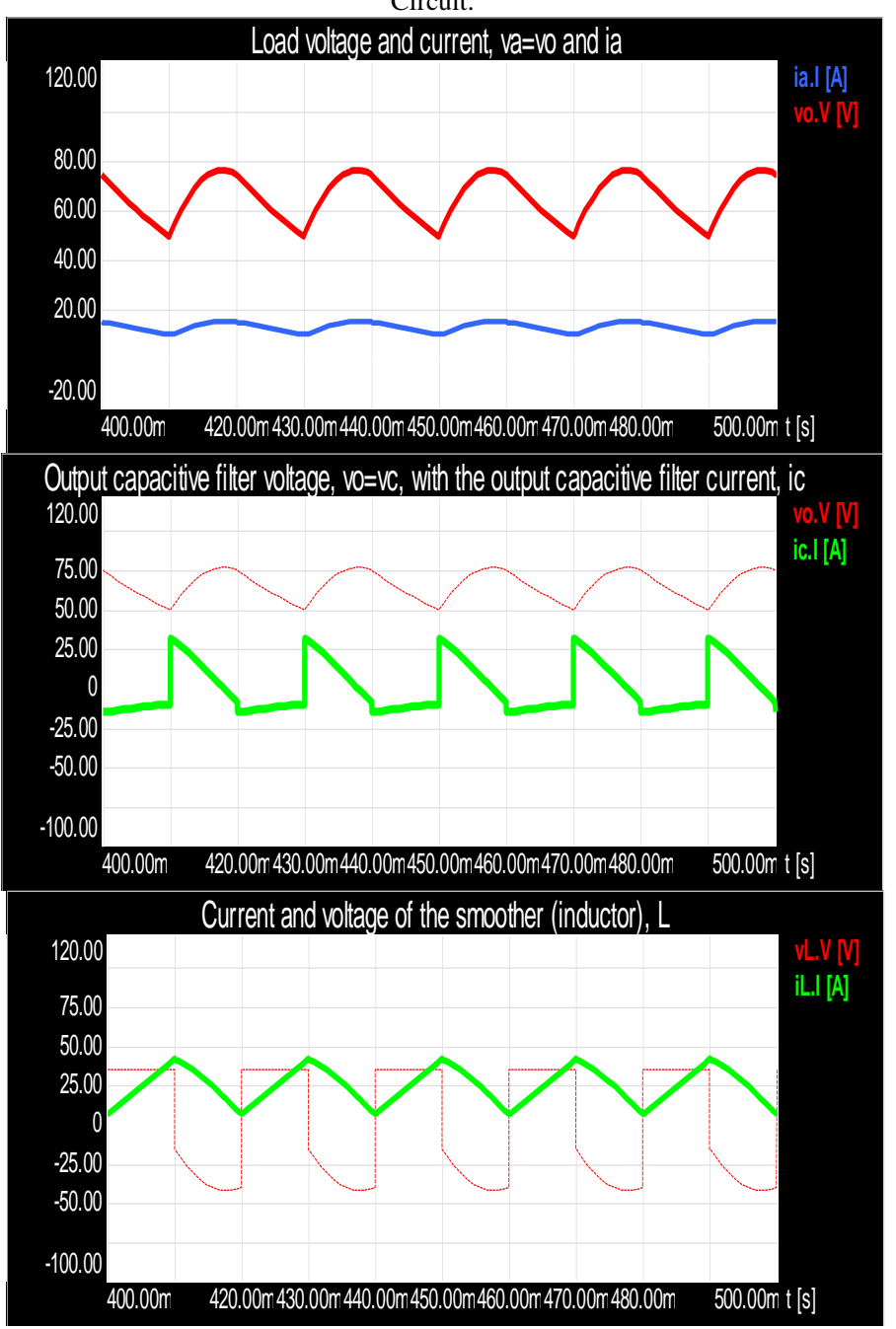

(b) Continuous Voltage and Current Waveforms in the basic Chopping up Regulator.

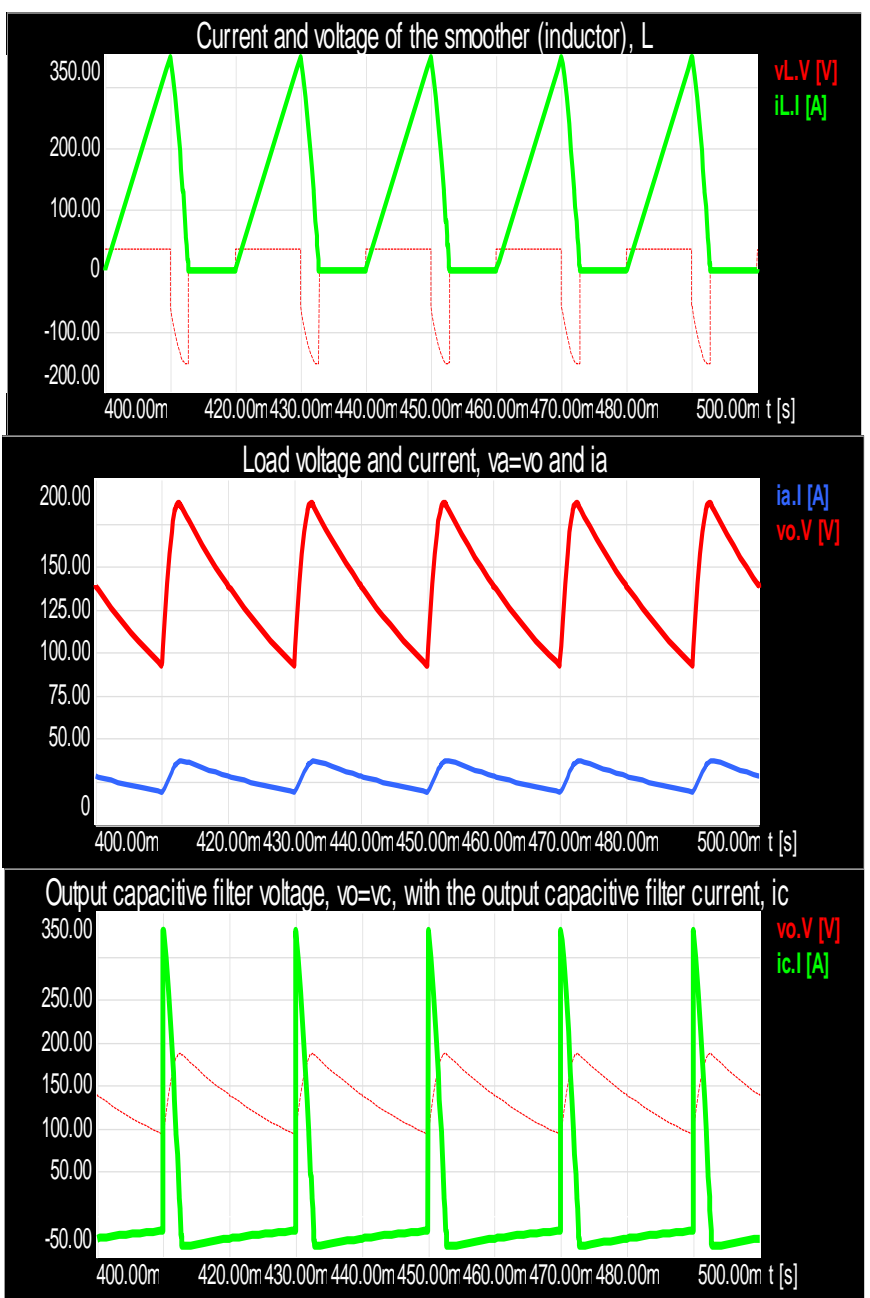

Fig. 1. (c) Discontinuous Voltage and Current Waveforms in the basic Chopping up Regulator.

The average value of the regulator output current, $i_{o}$, is the same as the average current in the load, thus:

$I_{\text {oavg }}=\frac{V_{o}}{R}=\frac{1}{2} I_{L \max } k_{1}=\frac{1}{2} \frac{V_{S} k}{f L} k_{1}$

After solving, it results in:

$k_{1}=\frac{V_{o}}{V_{S}} \frac{2 f L}{R k}$

Substituting for $k_{l}$ into Eq. 8, results in the following value for the average load voltage, $V_{o}$ within the discontinuous current regime:

$V_{o}=\left[\frac{1}{2}\left(1+\sqrt{1+\frac{2 k^{2} R}{f L}}\right)\right] V_{s}$

The boundary operating conditions between continuous and discontinuous current regimes occurs when the interruption and zero value of the smoother current occurs exactly at $t_{k}=\mathrm{T}$. The average value of the smoother current on the limits of discontinuous current regime may be determined as follows:

$I_{\text {flim }}=\frac{V_{s}}{2 f L}\left(1-\frac{V_{s}}{V_{o}}\right)$ 
The maximum values of limits (13) occur when $\boldsymbol{V}_{\boldsymbol{o}}$

$I_{\text {flimmax }}=\frac{V_{s}}{2 f L}$

The average value of discontinuous smoother current within the region of discontinuity is obtained in [1] as:

$I_{\text {Ldisav }}=\frac{k^{2} V_{o}\left(\frac{V_{o}}{V_{s}}-1\right)}{2 f L}=\frac{\left(1-\frac{V_{s}}{V_{o}}\right)^{2}\left(\frac{V_{o}^{2}}{V_{s}}-1\right)}{2 f L}$

From the above mentioned expression 13, it may be determined the smoother current curve at the boundary of discontinuous currents shown in Fig. 5.

- Advantages

The fundamental chopping up regulator is a low cost regulator with a simple topology that can be easily regulated and is able to provide high gains. The gating signals of the switch can be generated with well-developed integrated circuits or microprocessors. In order for the fundamental chopping up regulator to work properly it must get a smooth input current. High efficiency can be achieved with medium and low switching times.

- Disadvantages

The circuit components are not ideal (voltage drops on power electronic devices, capacitor series resistances, smoother resistances, switching losses) and the output is limited. The off time of the switches should be limited to below 0.90 or 0.95 in order to avoid short circuiting the switch and the dc source. Also as the switching time gets closer to unity, the output voltage becomes more sensitive to any changes in this time. This can make it to be more difficult to adjust the output voltage at higher gains. The switch must also have a high voltage rating due to having the output voltage across it when it is off. This sometimes requires a switch that will have a slower switching time, or have a higher forward resistance.

\section{CONVENTIONAL Two-Leg ChOPPING UP REgUlatoR WITH MAGNETICALLY UNCOUPLED INDUCTIVE SMOOTHER}

Fig. 2 depicts a circuit diagram for a conventional two-leg connection of chopping up regulator with magnetically uncoupled inductive smoothers $\mathrm{L}_{1}, \mathrm{~L}_{2}$. Such a chopper up has two legs connected in parallel, $\mathrm{S}_{1}, \mathrm{D}_{1}$ and $\mathrm{S}_{2}, \mathrm{D}_{2}$. They are supplied via a single phase bridge diode rectifier and are switched on non-simultaneously with the displacement time $\mathrm{T} / \mathrm{n}$. The load comprises a smoother $\mathrm{L}_{\mathrm{a}}$ and resistor $\mathrm{R}_{\mathrm{a}}$.

Inductive smoothers $\mathrm{L}_{1}$ and $\mathrm{L}_{2}$ are passive electrical elements (known as magnetically uncoupled filters) wounded from a wire coil around a magnetic core of steel to confine its magnetic flux within the total number of coil turns. They are designed to create magnetic fields in the core as a result of flowing current and induced voltages across their terminals. This leads to a considerable stronger magnetic induction than would be produced by a simple wire coil without a magnetic core.

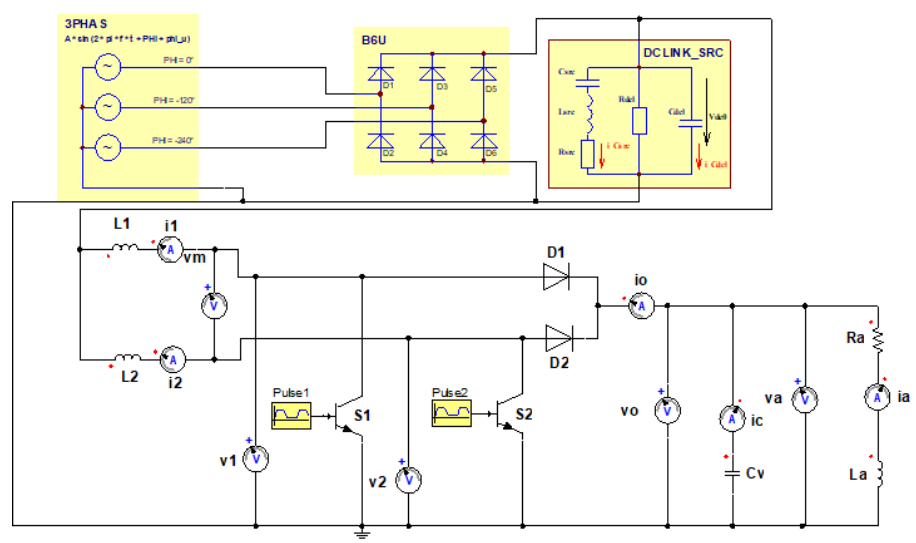

Fig. 2. Two-Leg Chopping up Regulator with Magnetically Uncoupledinductive Smoothers.

\section{A. Peak to Peak Current Ripple}

Traditionally, the several-leg chopping up regulator with magnetically uncoupled inductive smoother, as presented in Fig. 2, highly reduces the total current fluctuating waveforms flowing into the output capacitors and significantly the power increase as compared to the basic structure of the chopping up regulator. These results are listed for identical and linear design conditions of the inductive smoothers while $\mathrm{L}_{1}=\mathrm{L}_{2}=$ L. Therefore, the averaged currents into both legs are divided into their inductive smoothers, $\mathrm{L}_{1}, \mathrm{~L}_{2}$ equally by soft control of every leg main switch with a time displacement of $\mathrm{T} / 2$.

\section{B. Continuous Operating Regime}

Under the condition of a continuous conduction regime of operation, the two-leg regulator is simulated for the same parameters mentioned in Table I, whereas both smoothers have same inductance values, $L_{1}=L_{2}=L=30 \mathrm{mH}$. The voltage and current waveforms of the system for $\mathbf{0} \leq k \leq 1$ / 2are shown in Fig. 3.

The second area of economic interest where consumption increases are the loss of electricity due to transmission and distribution of electricity to end users. Technical losses that are not caused by human causes can be divided into losses in the lines and losses in voltage transformation. It can be seen from formula (1) that line losses are directly proportional to the quadrant of the maximum current, so the effort to control consumption is therefore to limit the peak of the load during the day, especially morning and evening [17-18].

Considering the switching duty ratio as $=t_{o n} / T$, then the ripple $\Delta i_{L}$ of the phase current flowing into individual smoothers should be same whether the switch is on or off, and it is obtained as:

$$
\begin{aligned}
& T=t_{o n}+t_{o f f}=\frac{L \Delta i_{L}}{V_{S}}+\frac{L \Delta i_{L}}{V_{o}-V_{S}} \Rightarrow \Delta i_{L}=\frac{V_{S}\left(V_{o}-V_{S}\right)}{f L V_{o}}= \\
& \frac{k(1-k)}{f L} V_{o}=\frac{k V_{S}}{f L}
\end{aligned}
$$




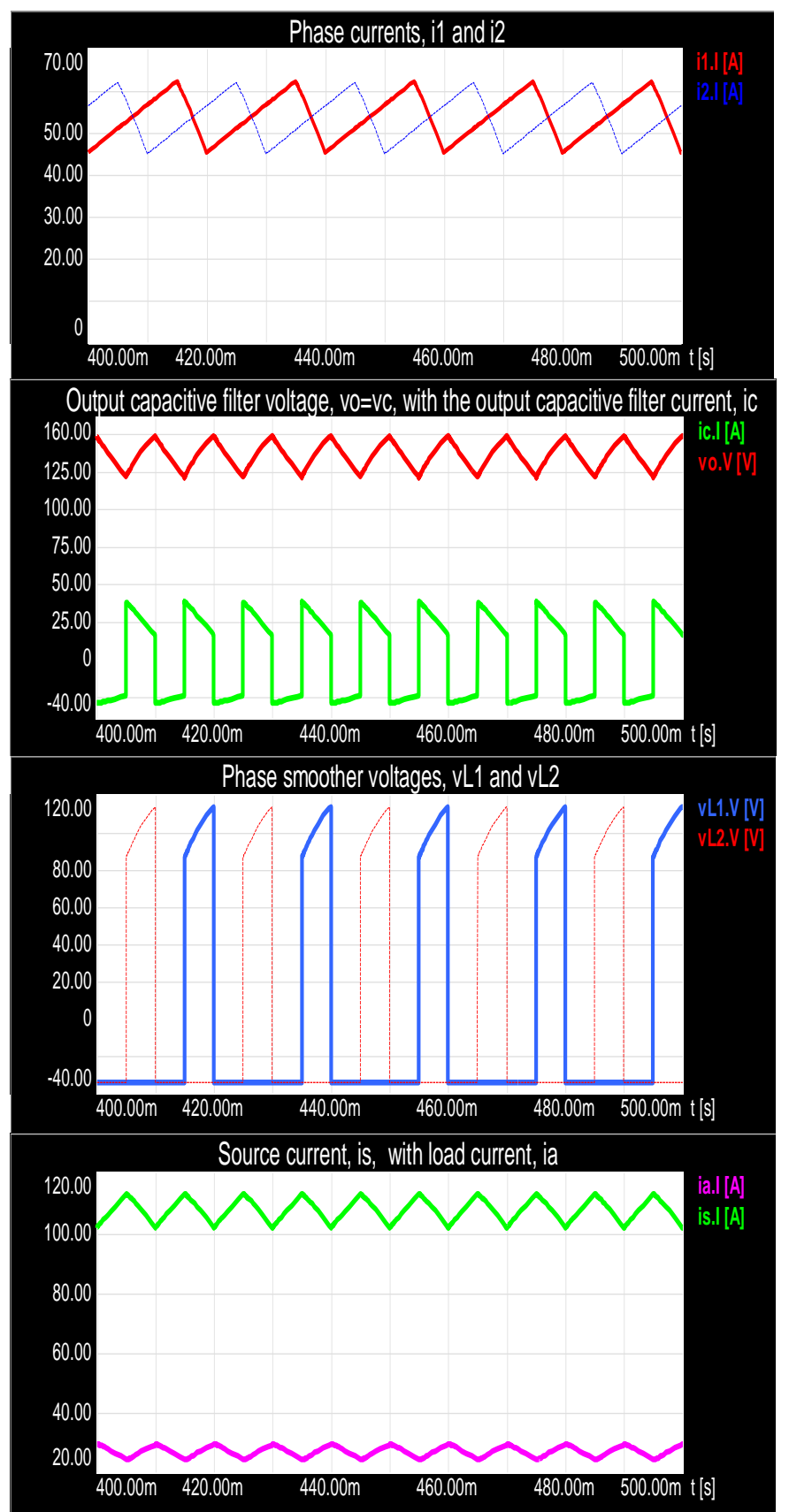

Fig. 3. Current and Voltage Steady State Waveforms of Two-Leg Connection of Chopping up Regulator with Flattering Inductive Smoothers for $0 \leq k \leq 1 / 2$.

The ripple of the source current $i_{s}$, may be determined for $\mathbf{0} \leq \boldsymbol{k} \leq \mathbf{1 / 2}$ from the sharp growth of this current during on-regime of one switch and off-regime of other switches. Thus, when switch $\mathrm{S}_{1}$ is on:

$\Delta i_{s}=\frac{V_{s}}{L} t_{o n}-\frac{V_{o}-V_{s}}{L} t_{o n}=\frac{2 V_{s}-V_{o}}{f L}\left(1-\frac{V_{s}}{V_{o}}\right)=$ $\frac{V_{o}}{f L}(1-2 k) k=\frac{V_{s}}{f L} \frac{(1-2 k) k}{1-k}$

The ripple of the source current in region $\frac{1}{2} \leq k<1$ is obtained using similar idea as in [9]:
$\Delta i_{s}=2 \frac{V_{o}-V_{s}}{L}\left(\frac{T}{2}-t_{o n}\right)=2 \frac{V_{o}-V_{s}}{f L}\left(\frac{1}{2}-\frac{V_{s}}{V_{o}}\right)=\frac{V_{o}}{f L}(3 k-$

$\left.2 k^{2}-1\right)=\frac{V_{s}}{f L} \frac{\left(3 k-2 k^{2}-1\right)}{1-k}$

The maximum value of the ripple of the source current occurs in region $\mathbf{0} \leq \boldsymbol{k} \leq \mathbf{1 / 2}$ when $\boldsymbol{k}=\mathbf{1 / 4}$ or when $V_{o}=\frac{4 V_{s}}{3}$ and in region $\frac{1}{2} \leq \boldsymbol{k}<1$ when $\boldsymbol{k}=3 / 4$ or when $\boldsymbol{V}_{\boldsymbol{o}}=\mathbf{4} \boldsymbol{V}_{\boldsymbol{s}}$, thus:

$\Delta i_{\text {smax }}=\frac{V_{o}}{8 f L}=\frac{V_{S}}{6 f L}$

The boundary between continuous and discontinuous current regime occurs as soon as the minimum value of the smoother current touches the zero axis exactly at $\boldsymbol{k}_{\mathbf{1}}=\mathbf{1}-\boldsymbol{k}$. Therefore, the average value of the phase smoother current flowing into each leg of the chopper up at the boundary of discontinuity is given exactly as in Eq. 13.

\section{Discontinuous Operating Regime}

In the previous analysis the switching frequency and the flattering smoothers of the regulator were considered to be sufficiently large and therefore, all currents in the circuit have normal continuous waveform. Otherwise, the source, phase and load currents are discontinuous as shown in Fig. 4.

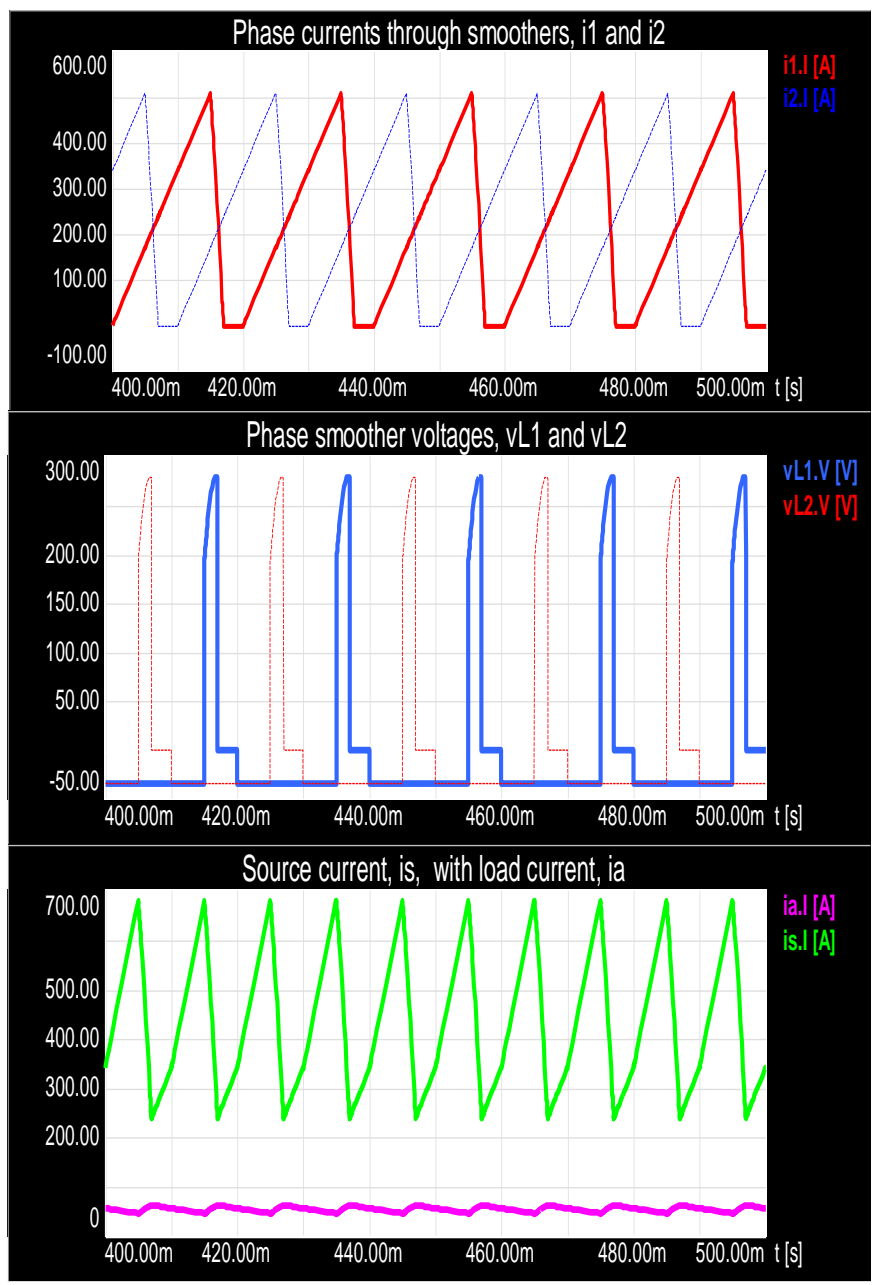

Fig. 4. Discontinuousphase Smoother Current Waveforms for $\mathbf{0} \leq \boldsymbol{k} \leq$ $1 / 2$. 


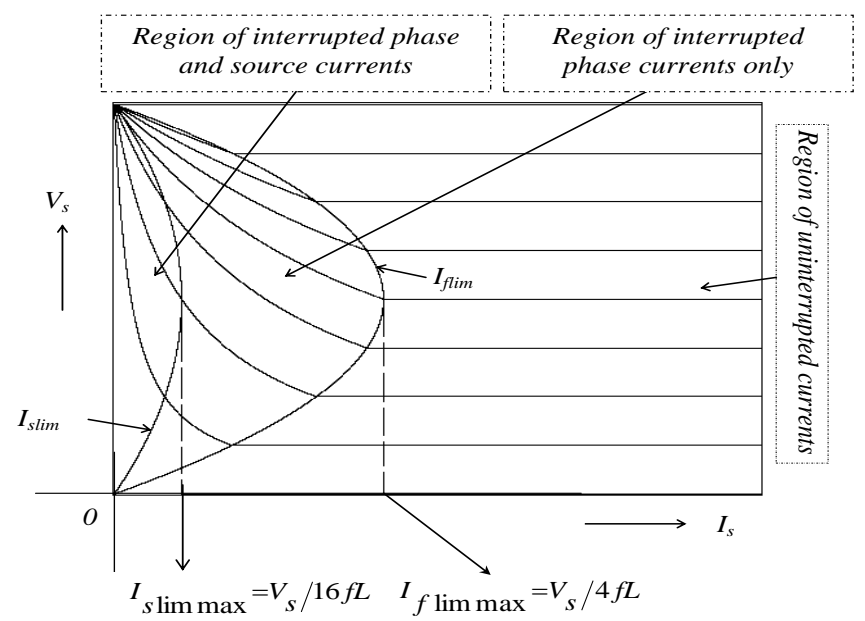

Fig. 5. Source Characteristics of the Regulator with the Border Curves in the Interrupted Region.

The average value of each smoother current within the region of discontinuity of phase currents has the same value as that in Eq. 15 which is obtained for the smoother current of fundamental connection. Therefore, the average value of the source current of the two-leg regulator within the region of discontinuity of phase currents is given as:

$I_{\text {sav }}=2 I_{\text {Ldisav }}=\frac{k^{2} V_{o}\left(\frac{V_{o}}{V_{S}}-1\right)}{f L}=\frac{\left(1-\frac{V_{s}}{V_{o}}\right)^{2}\left(\frac{V_{o}^{2}}{V_{S}}-1\right)}{f L}$

Eq. 20a determines the input current characteristics in the area of discontinuous phase and source currents shown in Fig. 5. If the source current is operating at the limits of discontinuity which may occur if and only if $\boldsymbol{k}_{\mathbf{1}}=\mathbf{1} / \mathbf{2}$, then the average value of such current at the boundary of the discontinuous source current region is determined as follows:

$I_{\text {slim }}=\frac{i_{L}\left(t_{o n}\right)}{2}=\frac{V_{S}\left(V_{o}-V_{S}\right)}{4 f L V_{o}}$

Where $\boldsymbol{i}_{\boldsymbol{L}}\left(\boldsymbol{t}_{\boldsymbol{o n}}\right)$ is the value of smoother phase current, $i_{L}$ at time $\boldsymbol{t}=\boldsymbol{t}_{\boldsymbol{o n}}$. The boundary between the continuous and discontinuous source current regions determined by relationship (21) is indicated in Fig. 5.

$\Rightarrow I_{\text {slimmax }}=\frac{V_{S}}{8 f L}=\frac{V_{o}}{16 f L}$

Eqs. 21 and 22are used to determine the input characteristic curves that describe the behavior of the currents within the region of discontinuity as shown in Fig. 5.

The peak to peak current ripples of the basic and two-leg chopping up regulators are shown as a function of the duty ratio, k in Fig. 6. Eqs. 6 and 19 have a good practical meaning because they are used to make an approximate calculation of the source current ripple slightly, which helps in determining the desired inductive smoother design values by selecting the appropriate ripple in it. From Fig. 6 It can be seen that this fluctuating ripple decreases with the number of legs of the regulator.

It should be noted that the number of inductive smoothers for $\mathrm{n}$-leg connection is $\mathrm{n}$ smoothers, but compared to the simple regulator each smoother is adjusted to a value of $1 / n$ of the source current. This advantage results in the use of smaller inductive smoothers on the output of the regulator in order to meet the same requirements for the total current ripple. Therefore, the distortion in the output voltage can also be improved due to smaller output coils, resulting in using a smaller capacitance at the output [1-2].

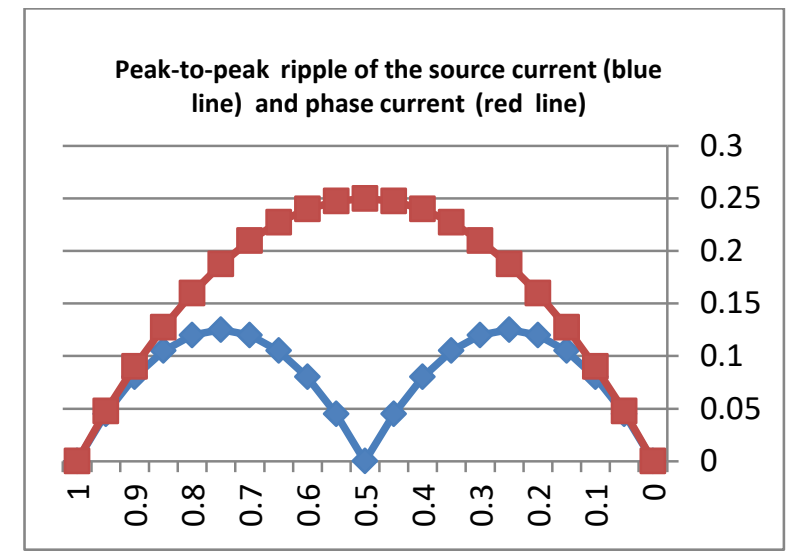

(a) Plot of Current Ripple of Source and Phase Current Versus Duty Time Ratio, $k$ as it Varies from Zero to Unity.

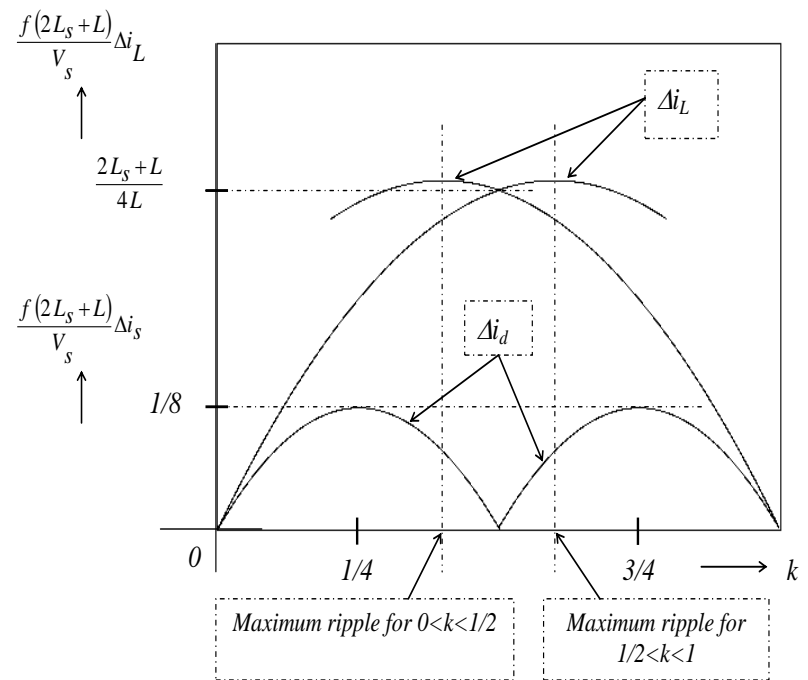

(b) Plot of Current Ripple of Source and Phase Current Versus Duty Time Ratio, $k$ with Additional Inductance at the Input Source $\mathrm{V}_{\mathrm{s}}$.

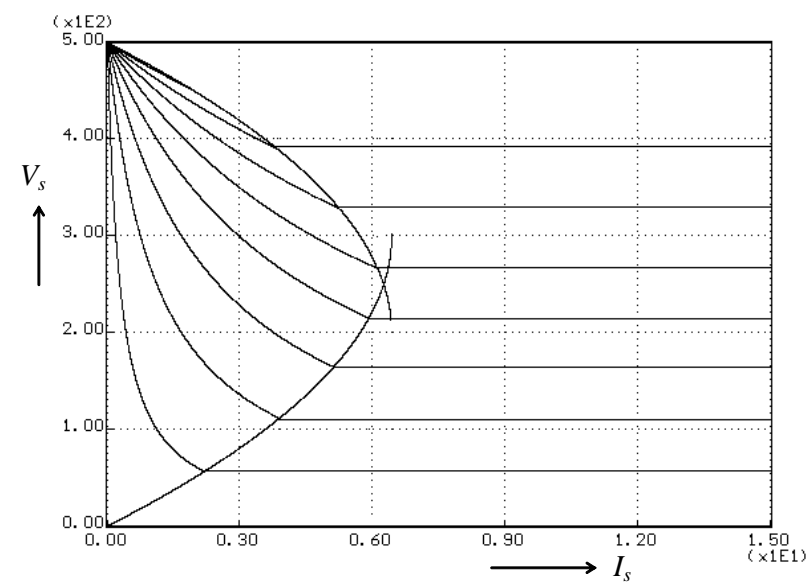

Fig. 6. (c) Plot of Source Current Curves Versus Source Voltage $V_{s}$ within the Region of Interrupted Currents. 
Inserting an additional inductance, $\mathrm{L}_{\mathrm{s}}$ in series with the source $V_{s}$, will result into the following expressions and graphs for the peak-to-peak ripple of the source and phase currents for $0 \leq k \leq 1 / 2$ as follows:

$\Delta i_{L}=\frac{V_{S}\left(L+L_{S}\right)-L V_{o}}{L\left(2 L_{S}+L\right)} t_{\text {on }}=\frac{V_{S}\left(L+L_{S}\right)-L V_{o}}{f L\left(2 L_{S}+L\right)} k$

Similar expressions may be obtained for $1 / 2 \leq k \leq 1$. Fig. 6(b) illustrates that the peak-to-peak smoother current ripple reaches its maximum value beyond its operating region at $=\frac{L_{S}+L}{2 L}>1 / 2$, therefore we consider its maximum value obtained at $k=1 / 2$ as shown in the figure. Fig. 6(c) presents the waveforms of the source and phase interrupted currents after adding an inductance at the input:

$I_{\text {sav }}=2 I_{\text {Ldisav }}=\frac{\boldsymbol{V}_{\boldsymbol{s}}\left(\frac{\boldsymbol{L}_{S}+\boldsymbol{L}}{\boldsymbol{L}}-\boldsymbol{k}\right) \boldsymbol{k}}{f\left(2 L_{S}+L\right)}$

\section{Two-LEG REGULATORS WITH INTERPHASING CENTRE- TAP TRANSFORMER}

Finally, complete content and organizational editing before formatting. Please take note of the following items when proofreading spelling and grammar:

In recent years, most power and regulation researches have been focused on the use of several-leg chopping up regulators to improve power electronic processors performance and power factor for personal computers, office equipment, space systems, laptops and telecommunication equipment as well as motor drive and power systems.

In addition, demand for electronic power processors for renewable energy systems has increased, since the improvement and growing production of industrial and commercial energy products and technologies and the growing dilemma of fossil fuels as a source of primary energy sources have expanded [10-14].

In general, the basic structure of the chopping up regulator topology with a single inductive smoother gives an acceptable performance in some of the aforementioned applications, but in some others such as distributed power conversion systems and power factor correction circuits, the performance of this configuration is enhanced by adding one or more legs with magnetically coupled smoothers that are operated in parallel or in series.

A connected coil is a device that is primarily used to store energy during the regulator switching duty cycle. The power entering the coupled coil is not the same as the power leaving it in a given instant. Coupled flattering inductive smoother are used to reduce regulator volume by using one core instead of two or more, to improve regulation of power regulators [2-5].

The aim of the work is to improve the performance of chopping up regulator by means of several-leg connection with directly coupled flattering inductive smoothers known as interphasing centre-tap transformer [2, 3, 12].

\section{A. Peak to Peak Ripple}

Fig. 7 shows the schematic diagram of the two-leg chopping up regulator with directly coupled flattering inductive smoothers. The coupled flattering inductive smoothers $L_{1}$ and $L_{2}$ share the same winding directions.

This type of regulators analysis can be best explained under a condition of a very small value of leakage inductances. Then, the windings of the two inductive smoothers have identical numbers of turns $N$. The inductance of each coupled flattering smoother is divided into two main parts [13-16]:

$L_{1}=L_{r 1}+L_{m}$

$L_{2}=L_{r 2}+L_{m}$

$L_{m}=\mu \sqrt{L_{1} L_{2}}$

Where $L_{1}, L_{2}$ are the self inductances of the flattering smoothers, $\mu$ is the magnetic coupling coefficient, $L_{r 1}, L_{r 2}$ are leakage inductances of the two flattering inductive smoother, $L_{m}$ is mutual inductance. In order to simplify the analysis, let's consider the inductive smoothers to have the same inductance values, $L_{1}=L_{2}=L$ and $L_{r l}=L_{r 2}=L_{r}$.

The aim of the work is to improve the performance of several-leg regulators by means of a several-leg parallel connection with an interphasing centre-tap transformer [2, 3, $6]$.

Concerning the switching technique of such regulator, whenswitch. $\mathrm{S}_{1}$ is on and switch $\mathrm{S}_{2}$ is off, $i_{1}$ flows into $\mathrm{S}_{1}$ and a similar large current $i_{2}$ flows into $D_{2}$ because of the magnetic coupling, and each leg carries approximately half the source current continuously and substantially equal voltages across the two halves of the winding. On the other side, when both switches are off, both currents will decrease exponentially into the two individual legs of the regulator. Therefore, there will be no need for controllers to distribute the currents equally into both individual legs as in the case of connection with smoothing inductive smoothers without magnetic coupling [1, $14]$.

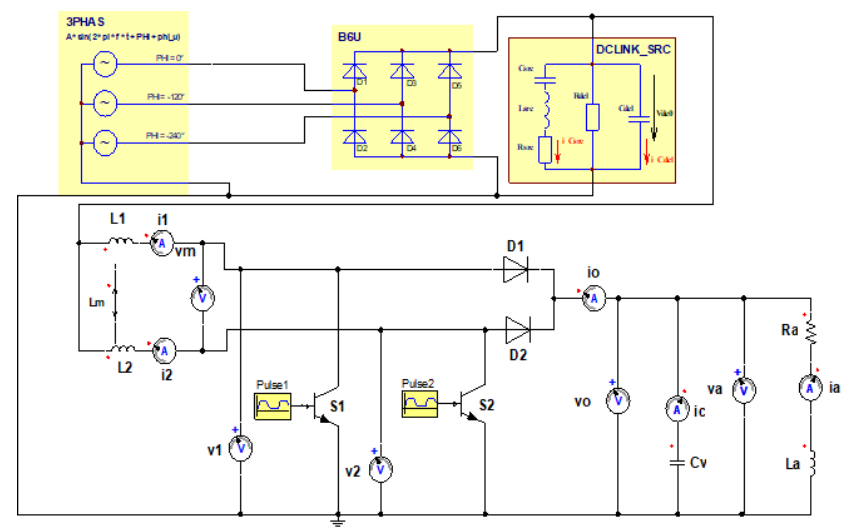

Fig. 7. Two-Leg Chopping up Regulator with Directly Coupled Flattering Inductive Smoothers. 


\section{B. Continuous Operating Regime}

The regulator simulation parameters for the continuous current regime are listed in Table II.

TABLE II. TeChNicAl Parameters of THE Simulated REgulator.

\begin{tabular}{|l|l|l|l|}
\hline Parameter & Symbol & Real value \\
\hline $\begin{array}{l}\text { Transformer winding } \\
\text { inductances: }\end{array}$ & $L_{l}=L_{2}$ & $30 \mathrm{mH}$ \\
\hline Smoothing filter: & $L$ & $30 \mathrm{mH}$ \\
\hline Regulator operating period: & $T$ & $20 \mathrm{~ms}$ \\
\hline Supply voltage & $V_{s}$ & $35 \mathrm{~V}$ \\
\hline Load resistance: & $R_{\mathrm{a}}$ & $5 \Omega$ \\
\hline Load inductance: & $L_{a}$ & $2 \mathrm{mH}$ \\
\hline Output capacitive filter & $C$ & $5 \mathrm{mF}$ \\
\hline Magnetic coupling & $k$ & 0.5 \\
\hline
\end{tabular}

Thus, assuming ideal devices with zero voltage drop and that the voltages across the individual legs of the regulatorare $V_{1}$ and $V_{2}$, respectively, then it can be concluded that during the on-regime of switch $\mathrm{S}_{1}$ and off-regime of switch $\mathrm{S}_{2}$ :

$$
\begin{gathered}
V_{1}=0=V_{s}-L \frac{d i_{s / 2}}{d t}=V_{s}-L_{r} \frac{d i_{1}}{d t}-L_{m} \frac{d i_{m}}{d t} \\
V_{2}=V_{s}+L \frac{d i_{s / 2}}{d t}=V_{s}+L_{r} \frac{d i_{2}}{d t}-L_{m} \frac{d i_{m}}{d t} \\
\frac{i_{s}}{2}=i_{1}+i_{m}=i_{2}-i_{m}
\end{gathered}
$$

Identical expressions may be derived for the on-regime of $\mathrm{S}_{2}$ and off-regime of $\mathrm{S}_{1}$.

When both switches, $S_{1}$ and $S_{2}$ are off simultaneously, the regulator diodes $\mathrm{D}_{1}$ and $\mathrm{D}_{2}$ conduct the phase currents to the load, and therefore:

$V_{1}=V_{2}=V_{s}-L \frac{d i_{s / 2}}{d t}=V_{s}-L_{r} \frac{d i_{1}}{d t}-L_{m} \frac{d i_{m}}{d t}=V_{s}-$

$L_{r} \frac{d i_{2}}{d t}+L_{m} \frac{d i_{m}}{d t}$

The voltage and current waveforms are illustrated for the regime of non-simultaneous conduction of switches $\mathrm{S}_{1}$ and $\mathrm{S}_{2}$ in Fig. 8 for $0 \leq k \leq 1 / 2$. During on-state of $S_{1}$, it can be concluded that:

$\Delta i_{s}=\frac{2 V_{s}}{L} t_{\text {on }}$

And under the condition of large value of output capacitive smoother, then during the off-state of $\mathrm{S}_{1}$ :

$\Delta i_{s}=\frac{2\left(V_{o}-V_{S}\right)}{L} t_{o f f}$

To avoid any damage that may occur due to the short circuiting of the supply to the chopper up when the main switch is on and to fully damp the fluctuations that appear in the source current ripple, it is suggested to add a flattering smoother $L$ into the circuit in series with the main input supply, $V_{s}[2,15]$. Therefore, under steady state conditions, the source current ripple $\Delta i_{\mathrm{s}}$ will be determined from the steep rise respective from the steep fall of the source current during the on-time respective the off-time of switch $\mathrm{S}_{1}$. This fluctuating waveform for the regime of non-simultaneous conduction of switches in region $(0 \leq k \leq 1 / 2)$ is obtained as follows:

$T=t_{\text {on }}+t_{\text {off }} \Rightarrow \Delta i_{s}=\frac{V_{S}}{2 f L}\left(\frac{k-2 k^{2}}{1-k}\right)=\frac{V_{o}}{2 f L}\left(k-2 k^{2}\right)$

For the regime of simultaneous conduction of switches in region $(\mathbf{1} / \mathbf{2} \leq \boldsymbol{k} \leq \mathbf{1})$, the source current ripple $\Delta i_{\mathrm{s}}$ is given as follows:

$$
\Delta i_{s}=\frac{V_{s}}{2 f L}\left(\frac{3 k-2 k^{2}-1}{1-k}\right)=\frac{V_{o}}{2 f L}\left(3 k-2 k^{2}-1\right)
$$

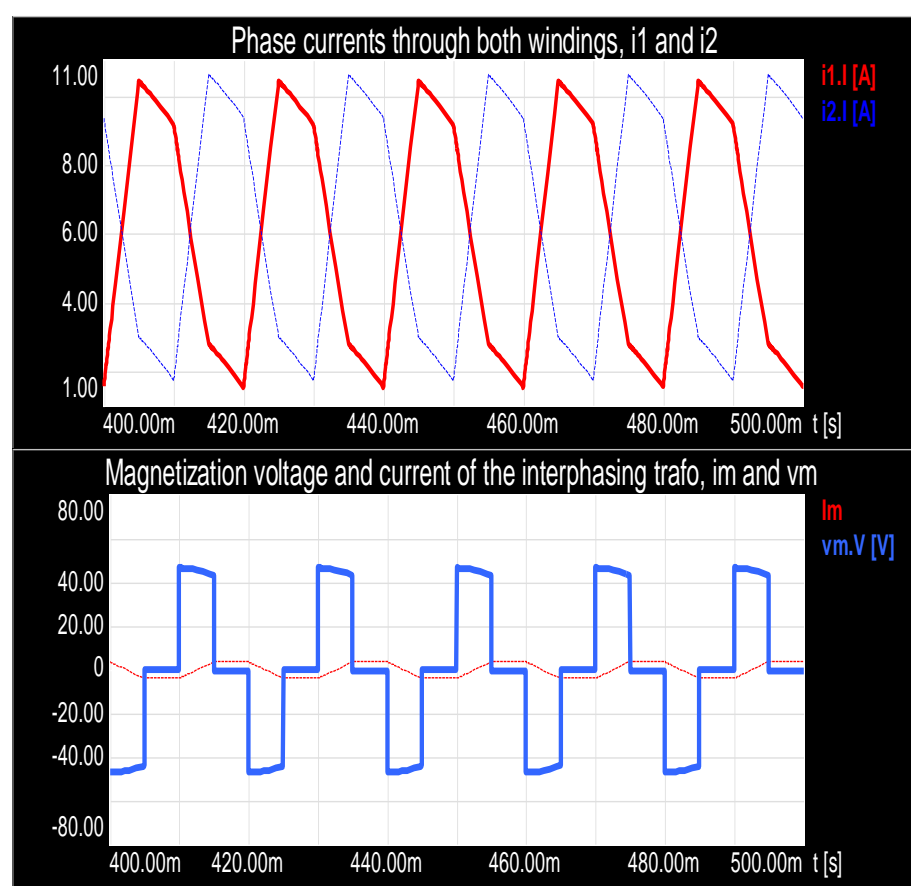

Outout capacitive filter voltage, $v 0=v c$, with the outout capacitive filter current, ic

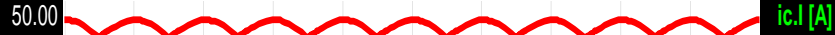

40.00

30.00

20.00

10.00

$-1000$

$-10.00$ $\begin{array}{llllll}400.00 m & 420.00 m & 440.00 m & 460.00 m & 480.00 m & 500.00 m \mathrm{~m}[\mathrm{~s}]\end{array}$

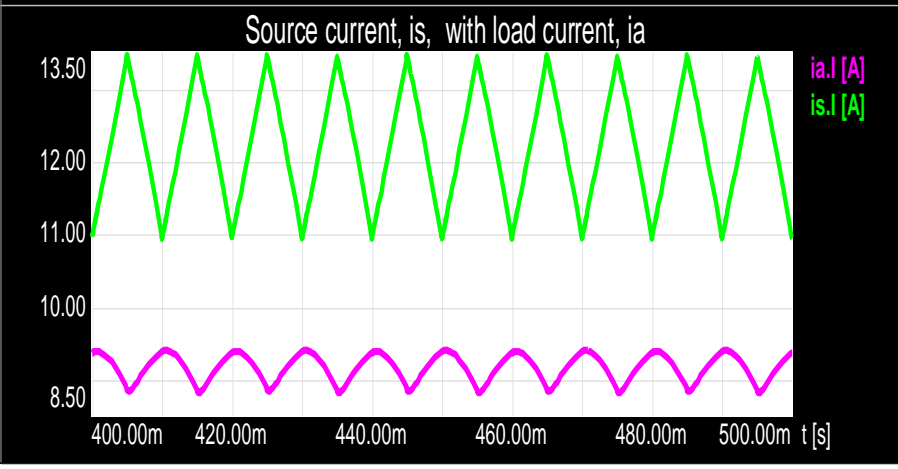

Fig. 8. Current and Voltage Steady State Waveforms of Two-Leg Chopping up Regulator with an Interphasing Centre-Tap Transformer for $\mathbf{0} \leq \boldsymbol{k} \leq \mathbf{1} / \mathbf{2}$. 
The maximum value of $\Delta i_{s}$ occurs in eq. 27 at $k=1 / 4$ and in eq.28at $k=3 / 4$. Thus:

$$
\Delta i_{\text {smax }}=\frac{V_{o}}{16 f L}
$$

Since the current rippling each phase is during the on-state of its active switches equal to the ripple in the off-state of this switch, then the maximum value of the magnetization current of the transformer occurs when $k=1 / 2$ as follows:

$$
I_{\mu \max }=\frac{V_{o}}{4 f L_{\mu}}
$$

Where $L_{\mu}$ is the magnetization mutual inductance between both smoothers of the transformer.

\section{Discontinuous Operating Regime}

In the previous analysis the smoother inductance was considered to be very large and due to the use of high frequency devices in the regulator, all currents in the circuit have normal continuous waveform and they do not even touch the zero-axis during the regulator operating period. But if the smoother has a small inductance and the switching frequency of the regulator is low, the source, the phase and the load currents are not sustained throughout the regulator operating period and they become zero before the end of this period as shown in Fig. 9.

The value of the inductance of smoothing smoothers is 20times smaller than that in the regime of continuous currents. Therefore, during the on-time of switch $\mathrm{S}_{1}$, the discontinuous phase current is expressed as follows:

$i_{1}(t)=\frac{V_{s}}{L} t$

At $\boldsymbol{t}=\boldsymbol{t}_{\boldsymbol{o n}}$ the phase current is transferred from switch $\mathrm{S}_{1}$ to its freewheeling diode $\mathrm{D}_{1}$ which means that:

$i_{1}(t)=i_{1}\left(t_{o n}\right)-\frac{V_{o}-V_{s}}{L}\left(t-t_{o n}\right)=\frac{V_{s}}{L} t_{o n}-\frac{V_{o}-V_{s}}{L}\left(t-t_{o n}\right)$

This conduction interval of $\mathrm{S}_{1}$ ends at the moment of interruption, $t=t_{k}$, when the phase current is distinguished.

This moment would be obtained from Eq. 33 by replacing $t$ with $t_{k}$ and putting $\boldsymbol{i}_{\boldsymbol{L}}\left(\boldsymbol{t}_{\boldsymbol{k}}\right)=\mathbf{0}$ :

$\boldsymbol{t}_{\boldsymbol{k}}=\frac{V_{o}}{V_{o}-V_{s}} \boldsymbol{t}_{\text {on }}$

Similar expressions could be obtained for all other phase currents of the regulator. The average value of the phase currents within the region of discontinuity is then obtained using Fig. 10(a) as:

$I_{a v 1}=\frac{i_{1}\left(t_{o n}\right) t_{x}}{2 T}=\frac{V_{o} V_{s}}{\left(V_{o}-V_{s}\right) f L} k^{2}$

The average value of the source current is then obtained as the sum of the average values of $\mathrm{n}$ phase currents, thus:

$I_{a v s}=n I_{a v 1}=n \frac{V_{o} V_{s}}{\left(V_{o}-V_{s}\right) f L} k^{2}$

Eq. 35 and 36 could be used to determine the input characteristics of the regulator that describes the behavior of the phase and source currents within the region of discontinuous operating regime. The variable parameter of such characteristics is the duty ratio, $\mathrm{k}$, whereas in this operating regime $\boldsymbol{V}_{\boldsymbol{s}} \neq(\mathbf{1}-\boldsymbol{k}) \boldsymbol{V}_{\boldsymbol{o}}$.

The boundary conditions for the phase currents at the limits of discontinuous operating regimes may be derived using the notations of Fig. 11 as:

$I_{\text {flim }}=\frac{V_{o}}{2 f L}(1-k) k$

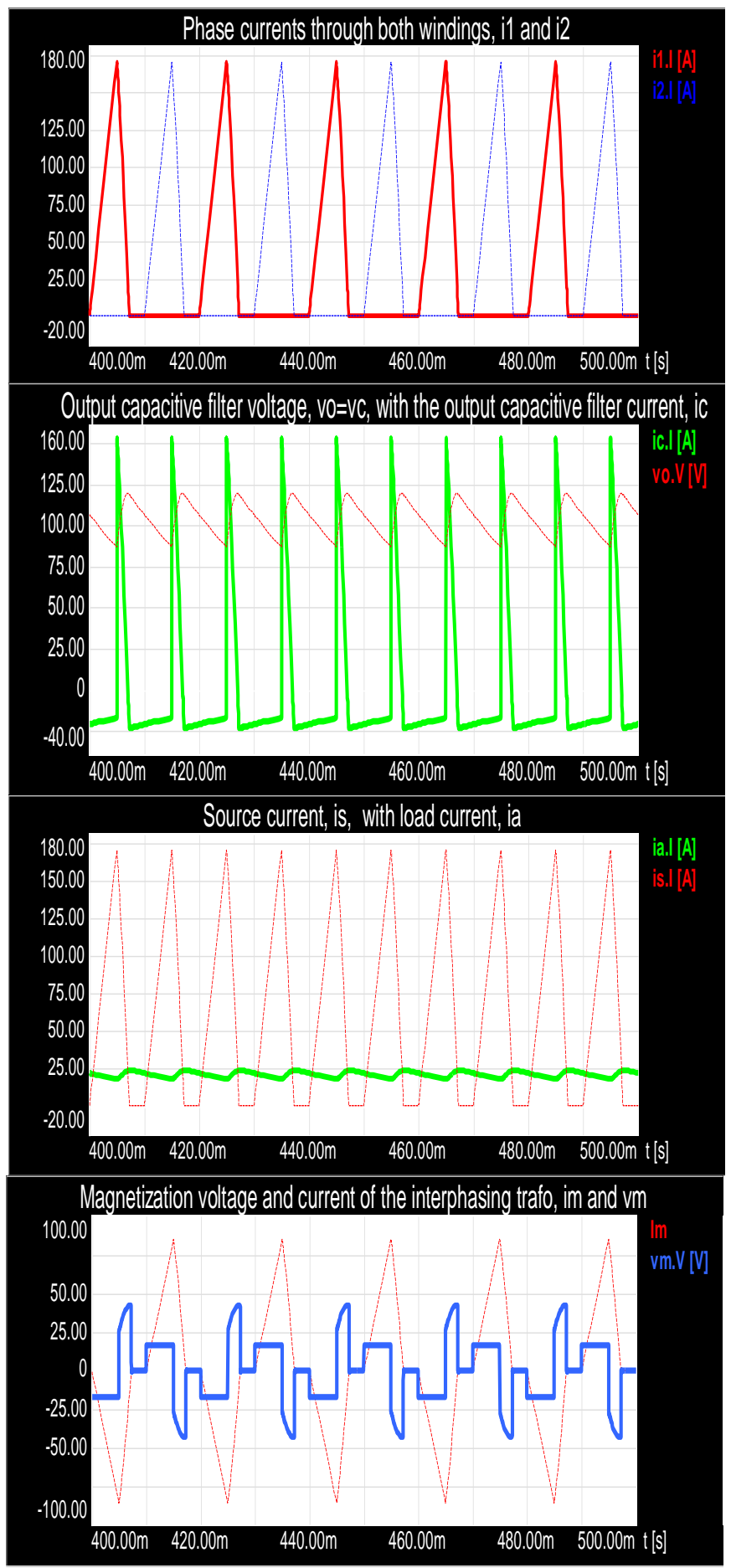

Fig. 9. Discontinuous Current Waveforms with an Interphasing Transformer for $0 \leq k \leq 1 / 2$. 
Hence, the regulator operates at the limits of discontinuous and continuous region of currents when the lower value of the smoother currents hits zero-axis exactly at the end of its conduction period. Using Eq. 37, the average value of the source current at the limits of discontinuity of phase currents is:

$I_{\text {slim }}=n \frac{V_{o}}{2 f L}(1-k) k$

However, if the discontinuous waveforms of phase currents overlap each other, as shown in Fig. 11, then the source current, $i_{s}$ would also have a discontinuous waveform. Therefore, the source current would touch the limits of interruption exactly at $\boldsymbol{t}_{\boldsymbol{k}}=\frac{\boldsymbol{T}}{2}$. Substitution this value for $\mathrm{t}_{\mathrm{k}}$ into Eq. 34, we get for the duty ratio the following value:

$\boldsymbol{k}=\frac{V_{o}-V_{s}}{n V_{o}}$

The boundary condition for the source current at the limits of discontinuity is then given as:

$I_{\text {slim }}^{*}=\frac{\Delta i_{1}}{2}=\frac{k V_{s}}{2 f L}$

Substituting from Eq. 39 into Eq. 40 yields:

$I_{\text {slim }}^{*}=\frac{V_{s}\left(V_{o}-V_{s}\right)}{2 n f L V_{o}}$

Similar expressions may be obtained for the source current in the conduction region of $\mathbf{0} \leq \boldsymbol{k} \leq \mathbf{1 / 2}$. The maximum unrestricted source current limit value is $I_{\text {slimmax }}^{*}=\frac{V_{o}}{32 f L}$. The source characteristic curves of the regulator with an interphasing transformer are illustrated in Fig. 10.

Adding an inductance, $L_{s}$, in series with the input source, $\mathrm{V}_{\mathrm{s}}$, results into the following expressions and waveforms for the source and smoother currents ripple at $0 \leq k \leq 1 / 2$ :

$\Delta i_{s}=\frac{2 V_{s}}{f L_{m}} k+\frac{V_{s}}{2 f L_{s}} k(1-2 k)$

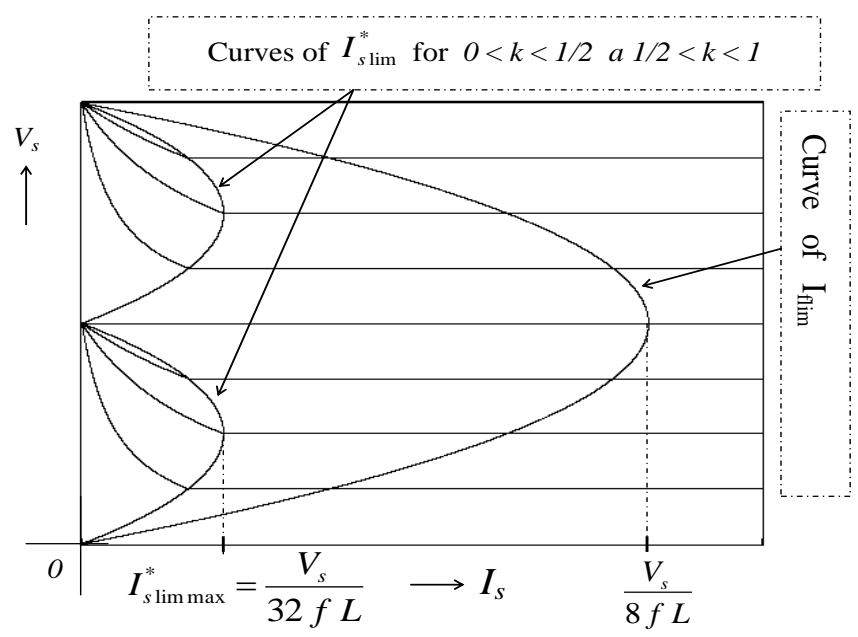

(a) Source characteristic curves with their borders in the region of interrupted currents.
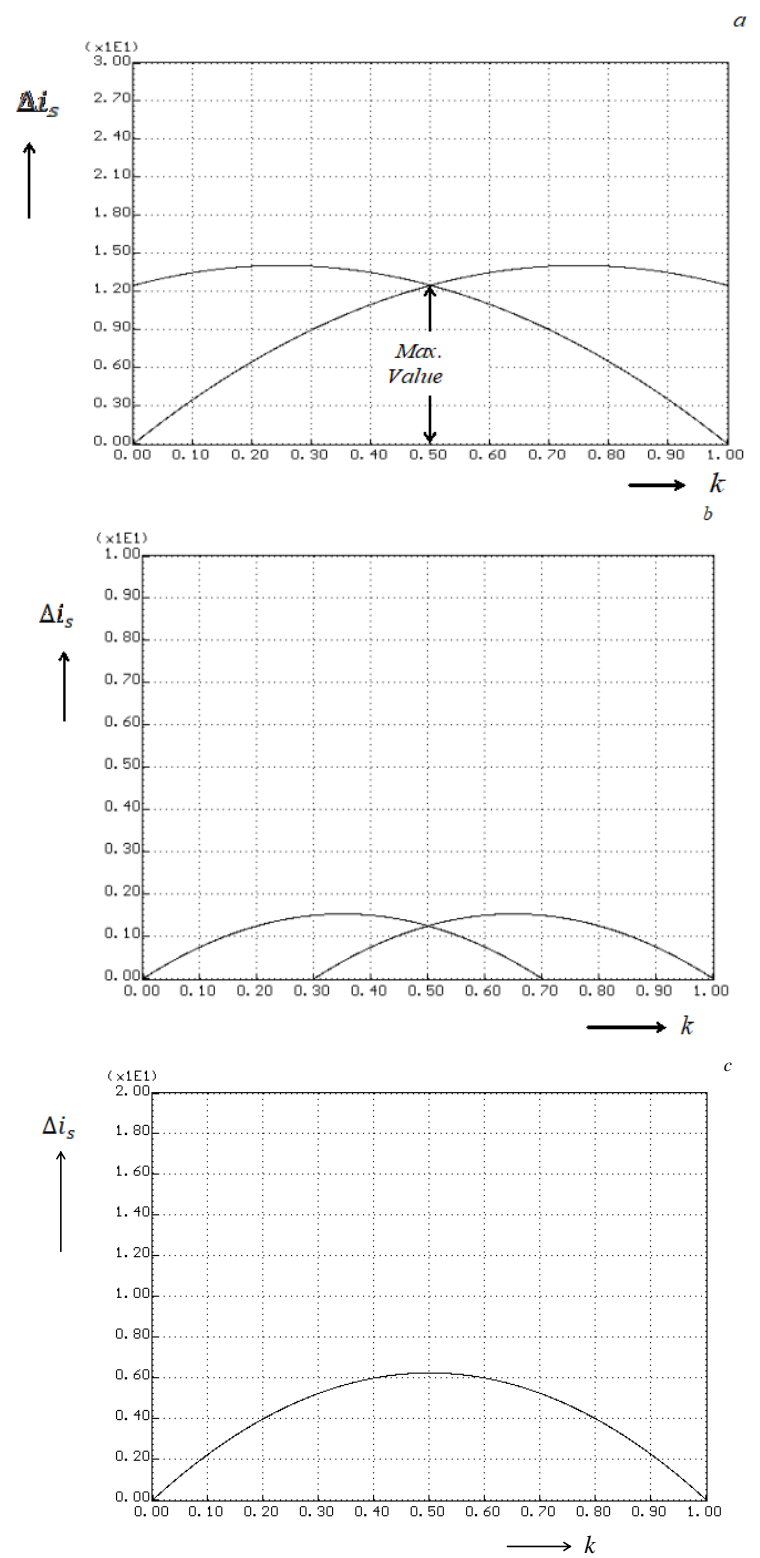

(b) Peak-to-peak ripple of source current with additional inductance at the input and different ratios: a) $L_{s}<4 L_{m}$, b) $L_{s}>4 L_{m}$, c) $L_{s}=4 L_{m}$

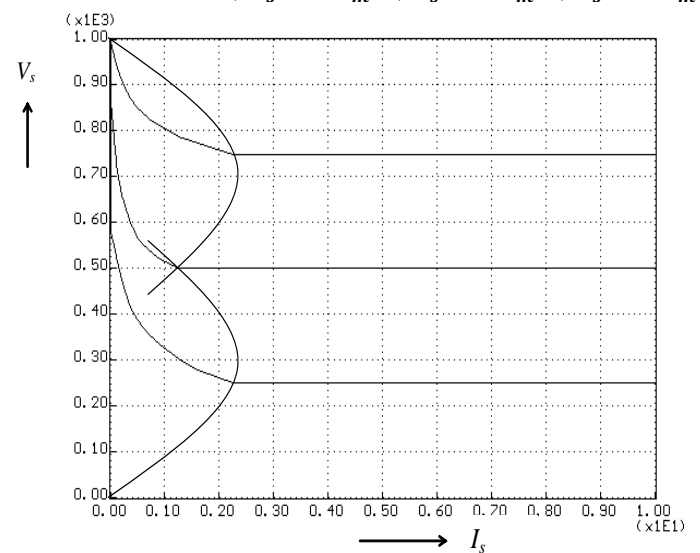

Fig. 10. Source Characteristic Curves with their Borders in the Region of Interrupted Currents for Real Values of $L_{s}$ and $L_{m}$. 
Maximum value for this ripple is obtained at $=\frac{4 L_{s}+L_{m}}{4 L_{m}}$. Since this switching ratio of maximum value is out of the first operating region of the converter, $\mathbf{0} \leq \boldsymbol{k} \leq \mathbf{1 / 2}$, the real maximum ripple is considered to be reached at $k=1 / 2$. Similar Expressions may be obtained for $\mathbf{1 / 2} \leq \boldsymbol{k} \leq \mathbf{1}$ :

$\Delta i_{s}=\frac{2 V_{s}}{f L_{m}}(1-k)+\frac{V_{s}}{2 f L_{s}}\left(3 k-2 k^{2}-1\right)$

Illustration of $\Delta \boldsymbol{i}_{\boldsymbol{s}}$ versus switching ratio $k$ for different values of the magnetization inductance $L_{m}$ and source inductance $L_{s}$ is shown below in Fig. 10(b). The average value of the source current at the limits of discontinuity within the operating region $\mathbf{0} \leq \boldsymbol{k} \leq \mathbf{1 / 2}$ is then given as $\Delta \boldsymbol{i}_{\boldsymbol{s}} / \mathbf{2}$. Fig. 10(c) presents the waveforms of the source and phase interrupted currents after adding an inductance at the input.

\section{Demands to Material of Flattering Inductive SMOOTHER AND TRANSFORMER}

Next issue will be to find a simple method for determining demands to materials of magnetically coupled inductive smoother to compare them with other connections of a chopping up regulator with a smoother. Taking into consideration a coil with a number of turns $N$, wrapped around a rectangular ferromagnetic core with a cross-sectional area $A$ and current $i$ flowing into it as shown in Fig. 11. Then, under the condition of linear core saturation curve and a neglected leakage flux, we can write for the inductance $L$ of the coil, the possible maximum value of the non-saturation magnetic flux $(\phi)$ density $B$ within the ferromagnetic core and the corresponding maximum value of the peak magnetization current $I_{\mu \max }$ that may flow within the magnetization inductance the following expression:

$\varnothing=N S_{F e} B=L I_{\mu \max }$

Concerning the coil, it is necessary to design its winding with respect to the maximum rms value of the smoother current, $\boldsymbol{I}_{r m s}$, that can flow through it. After rearrangement we can get [1]:

$$
\begin{gathered}
L I_{\mu m a x} I_{r m s}=N B S_{F e} I_{r m s}=N B S_{F e} \rho S_{\text {wire }} \\
=\rho B S_{F e} S_{c u}=\Sigma_{\text {material }}
\end{gathered}
$$

Wherein $\rho$ is the admissible density of a current flowing through a cylindrical coil wire of cross-sectional area $S_{\text {wire }}$, and $S_{c u}$ is the cross-sectional area of the whole winding of the coil with $\mathrm{N}$ turns and it may represent the material used in making the winding and $S_{F e}$ represents the material required for the design of the core [2].

The expression for demands to the materials of a simple inductive smoother for the fundamental connection (Fig. 1) may be simplified, if we put $\boldsymbol{I}_{\boldsymbol{L}}=\boldsymbol{I}_{\boldsymbol{r m s}}=\boldsymbol{I}_{\boldsymbol{\mu m a x}}$ where $\mathrm{I}_{\mathrm{L}}$ is the average value of the source current at the boundary of discontinuity. And thus:

$\boldsymbol{\Sigma}_{\text {material }}=\boldsymbol{L} \boldsymbol{I}_{\boldsymbol{L}}^{2}$

For the two leg connection without magnetic coupling we may get that:

$\Sigma_{\text {material }}=L \frac{I_{f}^{2}}{4}$

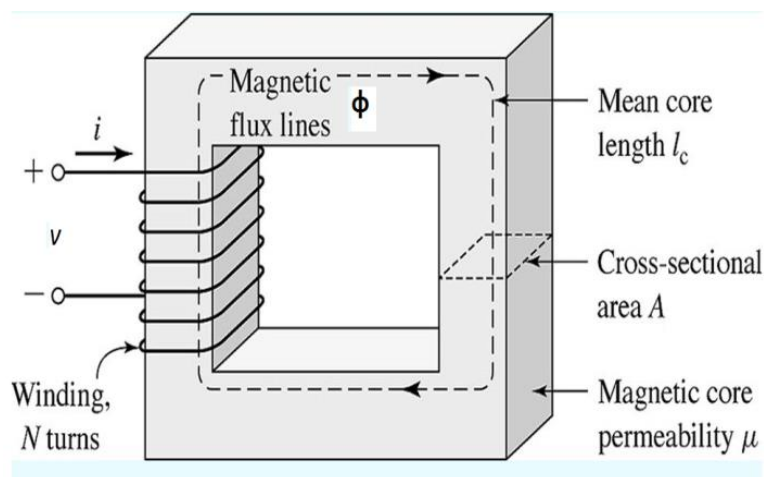

Fig. 11. Magnetic Core with a Coil.

Where $\boldsymbol{I}_{\boldsymbol{f}}=\boldsymbol{I}_{\boldsymbol{\mu m a x}}=\boldsymbol{I}_{\boldsymbol{r m s}}$ is the average current passing through each leg of the two-leg chopping up regulator.

Concerning the connection with an interphasing transformer, it may be concluded from the waveforms of the magnetization current, $i_{m}$ and magnetization voltage, $v_{m}$ in Fig. 9, that:

$$
\begin{gathered}
I_{\mu \max }-I_{\mu \min }=2 I_{\mu \max }=\frac{v_{m}}{L_{m}} t_{o n} \\
I_{\mu \max }=\frac{v_{m}}{f L_{m}} \frac{k}{2}=\frac{V_{o}}{2 f L_{m}} k=\frac{V_{s}}{2(1-D) f L_{m}} k
\end{gathered}
$$

According to the results of the simulation shown, for example, in Fig. 9 and 10, the maximum value of the magnetization current, $i_{m}$ occurs when $k=1 / 2$, therefore:

$I_{\mu \max }=\frac{V_{S}}{2 f L_{m}}$

Thus

$L_{m}=\frac{V_{S}}{2 f I_{\mu \max }}$

Substituting from Eq. 47 into Eq. 50 yields:

$\frac{L_{m}}{L}=\frac{V_{s} I_{r m s}}{2 f \Sigma_{\text {material }}}$

Eq. 51 represents the percentage of the magnetization mutual inductance within the overall inductance of each smoother which is used as the appropriate design of the inductive smoother and their common core.

For the design of the inductance of the uncoupled inductive smoother of a fundamental connection of boost regulator shown in Fig. 1 we may write:

$L=\frac{V_{o}}{4 f \Delta i_{\text {Lmax }}}$

The value of the inductance of one smoother in the case of two-leg chopping up regulator without magnetic coupling is given as:

$\boldsymbol{L}=\frac{\boldsymbol{V}_{\boldsymbol{o}}}{\mathbf{8 f \Delta \boldsymbol { i } _ { \text { smax } }}}$

Concerning the chopping up topology with an interphasing transformer, the inductance of the smoother connected directly to the output load is given as:

$\boldsymbol{L}=\frac{V_{o}}{16 f \Delta i_{\text {smax }}}$ 
The selection of the appropriate value for the inductance of a certain smoother should satisfy the critical inductance condition which happens when the current through the smoother decays to zero just prior to the next on time of the chopping up regulator switch.

\section{CONTROL TeChNiQues of Boost CONVERTER WITHOUT TRANSFORMER}

As it has been said, the distribution of currents into phases of two-leg chopper up regulator without magnetic coupling is achieved using suitable control techniques to proportionally adjust the switching ratio of each leg. Concerning the two-leg connection with magnetic coupling the interphase transformer takes care of distributing the currents equally into phases, and therefore there will be no need for controllers for this purpose [9].

The output voltage of the regulator is compared within a comparator with a reference voltage to produce an error signal. This error signal is then used to adjust the switching ratio of the regulator via a pulse modulator. An amplifier is then used to amplify the modulator output just to bring it to the required voltage and power level suitable for driving the chopper regulator [2].

The output voltage of the regulator can be changed by controlling the switching ratio $k$, and this may be using achieved Constant frequency method: The regulator, or switching, frequency $f$ (or chopping period $T$ ) is kept constant and the on-time $T_{l}$ is varied. The width of the pulse is varied and this type of control is known as pulse width modulation (PWM) control [1]. The PWM control circuit may be composed of a square wave oscillator, a flip-flop, an integrator and two gates.

The output voltage of the chopper regulator can be changed by controlling the switching ratio $k$, and this can be achieved by using the constant frequency method, where the switching frequency $\mathrm{f}$ of the regulator is kept constant and its on-time $t_{\text {on }}$ is varied. The pulse width of the triggering signal for each switch is therefore distinct based on the level of the required output voltage. This type of control technique is known as pulse width modulation control technique (PWM). The PWM control circuit usually comprises a square wave oscillator, flip-flop logic circuit, integrator, and two gates.

The frequency of the master square wave oscillator is chosen to be twice that of the regulator operating frequency. The triangular wave is generated by the help of an integrator which is the compared with an error signal to produce the pulse width modulation [13-14].

The current distribution equally into both legs of the regulator would be in accord with their respective forward voltage drops. Therefore, using one controller for both legs does not guarantee a same voltage drop across each leg and consequently a same average value of current through each phase. Therefore, it is advised to implement two PI controllers, one for each leg as it is shown in Fig. 12. Even though, these two controllers can help the equal distribution of currents average value but it does not help current sharing of their dynamic values [2].

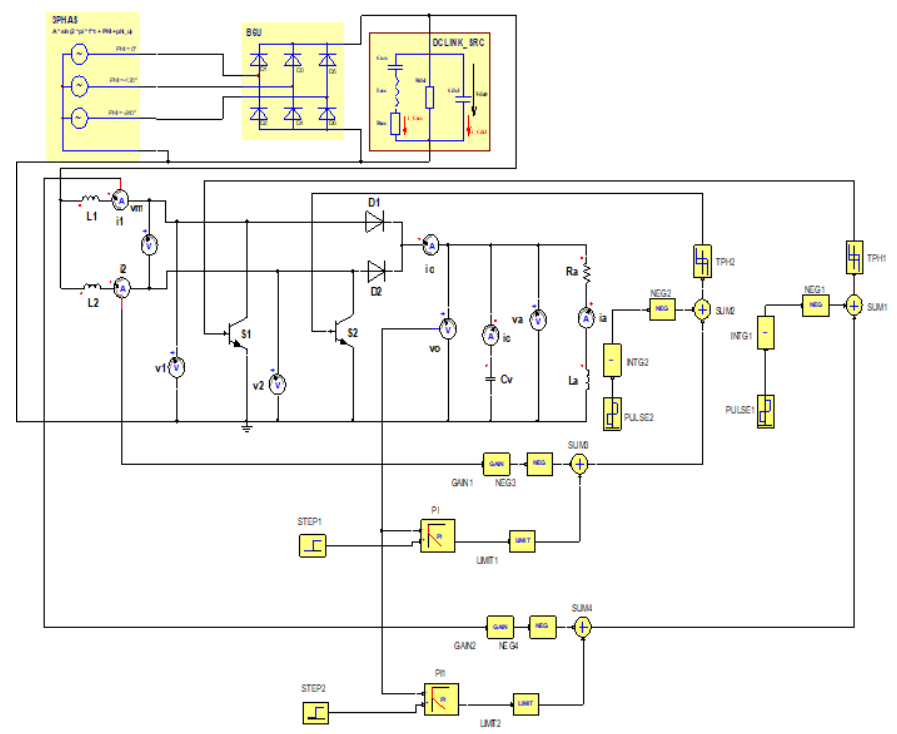

Fig. 12. Control Circuit for Two-Leg Chopper Regulator without a Transformer.

\section{SiMULATION RESULTS}

A two leg chopping up regulator with directly coupled flattering inductive smoother or with an interphasing centretap transformer is analyzed and simulated. All the components of the regulator including BJTs, diodes, coupled flattering inductive smoother are assumed to be ideal. They are replaced by prototype regime is of almost zero resistance in the onstate, approximately zero fall and rise switching time and infinite resistance in the off-state. Dynamic properties of the diodes and switches were ignored. It does not have a weighty effect on the main chopping up operation, particularly when the chopper up operating frequency is not closed to the cutoff frequency of the transistors.

Equations 36, 38 and 41 are the desired results of the analysis of the chopping up regulator since they represent the behavior of its input and phase currents in the discontinuous and continuous regions shown in Fig. 13.

This figure illustrates the source current peak-to-peak ripple of the chopping up regulator with fundamental connection, flattering inductive smoother, and with an interphasing centre-tap transformer. With magnetically uncoupled flattering inductive smoother, the maximum input current ripple is $0.125 \mathrm{~A}$. With direct coupling, the input current ripple is reduced to $0.0625 \mathrm{~A}$.

Wherein $\rho$ is the admissible density of the current flowing through a cylindrical coil wire of cross-sectional area $S_{\text {wire }}$, and $S_{c u}$ is the cross-sectional area of the whole winding of the coil with $\mathrm{N}$ turns.

Fig. 14 illustrates the decrease in the amount of material required for the transformer and filters design process and in the magnetization current as a result of the increase in switching frequency or strength of the magnetic coupling between its filters. 


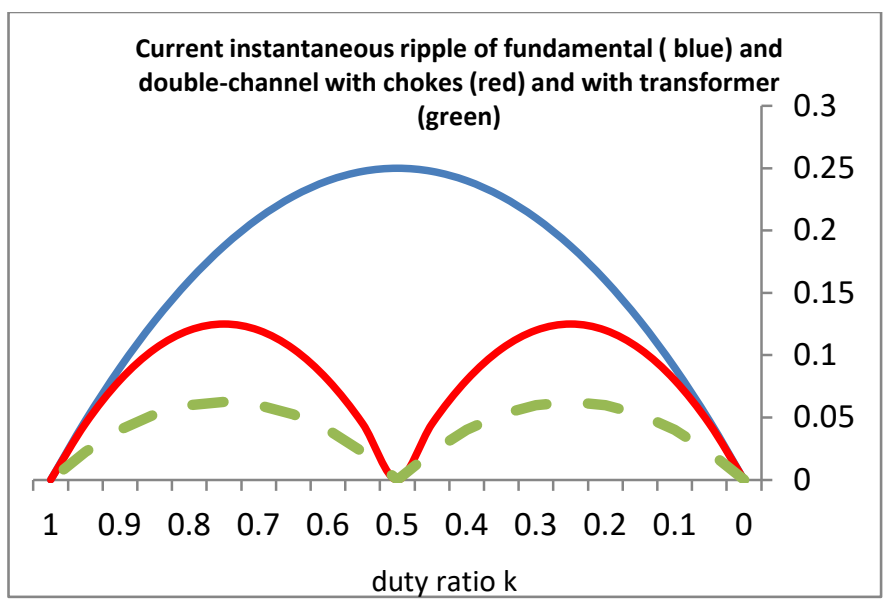

Fig. 13. Source Current Ripple Versus Duty Ratio.

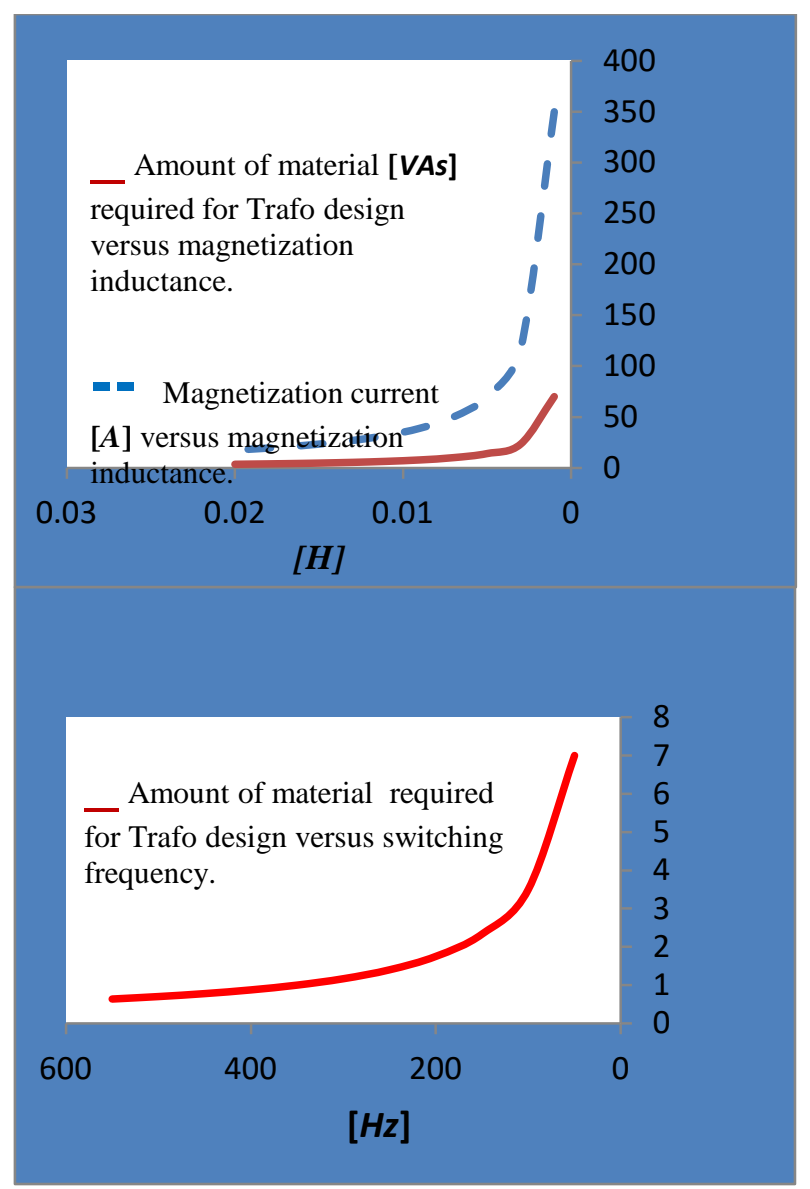

Fig. 14. Plot of Material Design Versus (a) Magnetization Inductance. (b) Switching Frequency.

\section{CONCLUSION}

This paper emphasis on the idea of design process, overall current and voltage reduction of two leg dc-dc chopping up regulators by using magnetically coupled flattering inductive smoother wrapped around a common core identified as an interphasing centre-tap transformer. Detailed analysis has been done, while simulation and experimental results have been done to validate the concept. In addition, it has been found that phase current fluctuating waveform will also be decreased as compared to the fundamental regulator connection and to the connection with magnetically uncoupled inductive smoother. This may improve the dynamic performance of the system and increase its efficiency. Furthermore, to obtain better results, magnetic coupling coefficient should be carefully chosen.

Using the interphase transformer with the chopper up regulator can help not only to accomplish both conditions of equal sharing of currents into individual legs (dynamic and steady-state) but also, as it has been said at the beginning of this research, it abolishes the need for using any controllers.

An interleaved two-leg chopping up regulating topology, however, improves regulator performance at the cost of additional smoothers, power electronic devices, and input rectifiers. Since the smoother is the largest and heaviest component in a power boost converter, the use of a coupled smoother, where a core is shared by multiple regulators instead of using multiple discrete smoothers, offers a potential approach to reducing the filters size, volume and weight. Coupled smoother topologies can also provide additional advantages such as reduced core and winding losses as well as improved input and output current and voltage ripple characteristics. The designer may choose to reduce either the boost smoother volume or increase the switching frequency to reduce the size of the EMI filter. In some cases just adding an additional phase will reduce the size of the EMI filter. Multiphase connection of such regulators also reduces the RMS current in the chopping up regulator capacitor greatly reducing electrical over stress on the capacitor. However, further study should be done to show if the complexity and cost of the design will increase with each additional phase.

\section{REFERENCES}

[1] Walid Emar, Khader Mohammad, and Mahdi Washhais: Multileg Step Up Power Converter with Magnetically Uncoupled Filters, (International Journal of Power and Energy Systems), ACTAPRESS, Vol. 36, No. 1, 2016.

[2] Issam Trrad,Walid Emar, Ziad Sobih: Novel Hybrid Two-Phase Interphase-Reactor Boost Type Converter with Controlled Output Voltage and Sinusoidal Input Utility Voltage, IJREEE, Vol. 3, issue 2, 2015.

[3] Muhammad H. Rashid. Power Electronics, Circuits, devices, and Applications, Electrical and Computer Engineering, University of West Florida, Pearson Education International, third edition, 2004.

[4] Khadmun W , Subsingha W. High Voltage Gain Interleaved DC Chopper up regulator Application for Photovoltaic GenerationSystem, Energy Procedia, ELSEVIER, 10th EcoEnergy and Materials Science and EngineeringSymposium, Volume 34, 2013; Pages 390-398.

[5] N. Mohan, T. M. Undeland, and W. P. Robbins, "Power Electronics, Converters, Applications, and Design," John Wiley \& Sons, 2003.

[6] Ying qiu, Helen Liu, and Xiyouchen: Digital Average Current regime control of PWM DC-DC Converters Without Current Sensor. In: IEEE Transactions on Industrial Electronics, May 2010, Vol. 57, pp. 16701677.

[7] Chander, S. Agarwal, P. and Gupta, I.: Auto-tuned, discrete PID controller or DC-DC converter for fast transient response. In: International Conference on Power Electronics, Dec 2011, pp. 1-7.

[8] Chuanlin Zhang, Junxiao Wang, Shihua Li, BinWu,and Chunjiang Qian.: Robust Control for PWM-Based DC-DC Boost Power Converters With Uncertainty Via Sampled-Data Output Feedback.In: IEEE Transactions on Power Electrionics, Jan 2015, Vol. 30, No. 1, pp. 504-515. 
[9] Ramesh kumar, K, Jeevananthan, S.: A Sliding Regime Control for Positive Output Elementary Luo Converter. In: Journal of Electrical Engineering,Nov 2010, Vol. 10, pp. 115-127.

[10] Subramanian Vijayalakshmi, and Thangasamy SreeRenga Raja.: Time domain based digital PWM controller for DC-DC converter. In: Automatika, Dec 2014, Vol. 55, No. 4, pp. 434 - 445.

[11] Rengamani Shenbagalakshmi, and Thangaswamy SreeRenga Raja.: Discrete prediction controller for DC-Converter. In: Acta Scientiarum. Technology Maringá, Jan 2014, Vol. 36, No. 1, pp. 41-48.

[12] Lung sheng yang, tsorng-juu liang ,hau-cheng lee, j. chen, “ Novel high step up DC-DC converter with coupled smoother and voltage doubler circuit" IEEE 2011 trans. Industrial electronicsvol 58.

[13] Yi-ping hsieh, jiann-fuh chen, tsorng-juu liang lung-sheng yang "Novel high step up DC-DC converter with coupled smoother and switchcapacitor techniques" IEEE 2012 trans. Industrial electronics vol 59.

[14] M. Jang, M. Ciobotaru, and V.G. Agelidis, "A Single-Phase GridConnected Fuel Cell System Based on a Boost-Inverter," IEEE Trans. Power Electronics. Appl., vol. 28, no. 1, pp. 279-289, Jan. 2013.

[15] F. Lin Luo and H. Ye "Power Electronics: advanced conversion Technologies" CRC Press, 2010.

[16] M. Amundarain, M.Alberdi, A.J. Garrido, and I. Garrido, "Regimeling and Simulation of Wave Energy Generation Plants: Output Power Control" IEEE Transactions on Industrial Electronics, Vol. 58, No. 1, Pp. 105-117, 2011.

[17] K.G. Remya, Chikku Abraham, and Babita R. Jose, " A Slope Compensated Current Regime Controlled Boost Converter",
Communications in Computer and Information Science, 2012, Volume 305, Part 3, 69-76.

[18] Mohan, Undeland and Robbins "Power Electronics: Converter Applications and Design" John Wiley \& Sons, ISBN, 978-0471226932, 2002.

\section{AUTHOR'S PROFILE}

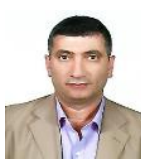

Walid Emar received his B.Sc. and his M.Sc. degree in power electronics in 1996/1997 and his Ph.D. degree in power electronics and control in 2002 from the University of West Bohemia, Czech Republic. Currently, He is at Isra University, Jordan as a full-time associate professor for teaching Energy Management for Master degree students and control systems, electrical machines, in addition to power electronics and other subjects for undergraduate students. He is also engaged in research in control of power electronics and machinery control.

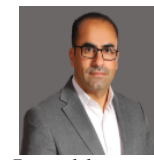

Omar A. Saraereh received his B.Sc. in telecommunication engineering from Mu'tah University, Jordan, in 1999, the M.Sc. degree in digital communication systems in U.K., and the Ph.D . degree in electrical and electronic engineering/mobile communications from Loughborough University, Loughborough, U.K., in 2005. He is currently an Associate Professor with the Department of Electrical Engineering, The Hashemite University, Jordan. He has published many papers in various international journals and conferences. 